\section{Sex Pheromone of Rice Stem Borer; Purification and Chemical Properties}

\author{
Kyuji Ohta, Sadahiro Tatsuki, \\ Kyoichi UCHIUMI, Masaaki KurIHARA \\ and Jun-ichi FuKaMI \\ The Institute of Physical and Chemical Research, \\ Wako-shi, Saitama 351
}

Received September 12, 1975

The female moths of the rice stem borer, Chilo suppressalis Walker, a serious pest of rice plant, produce sex pheromone which lures the male moths and elicits mating dance prior to mating." ${ }^{\prime \prime}$ This introductory report deals with purification and chemical properties of the sex pheromone. The pheromonal activity was followed by laboratory bioassay method based on mating dance of the male moths. ${ }^{2}$

The crude extract of the pheromone was prepared by immersing whole bodies of about 7000 virgin female moths* in 1,2-dichloroethane and concentrating the solution in vacuo after drying over anhydrous sodium sulfate. On preliminary experiments, saponification of the crude extract failed to destroy the activity, which suggested the absence of an ester group. When the crude extract was chromatographed on a column of Florisil moistened with water $(7 \%)$ and the column was successively eluted with hexane, $5,15,50 \%$ ether in hexane, and ether, $5 \%$ ether in hexane removed active material, which suggested that the pheromone had less polar group than an alcoholic group. The latter is expected to be eluted by $15 \%$ ether in hexane. ${ }^{3)}$ These indicated that the pheromone of this species had no common functional groups of known lepidopterous sex pheromones, which were mostly long straight chain alkenols, alkadienols or their acetates. $^{4)}$

For purification of the crude extract, an oil

* The insect was successively reared in this laboratory with rice seedling as diet.

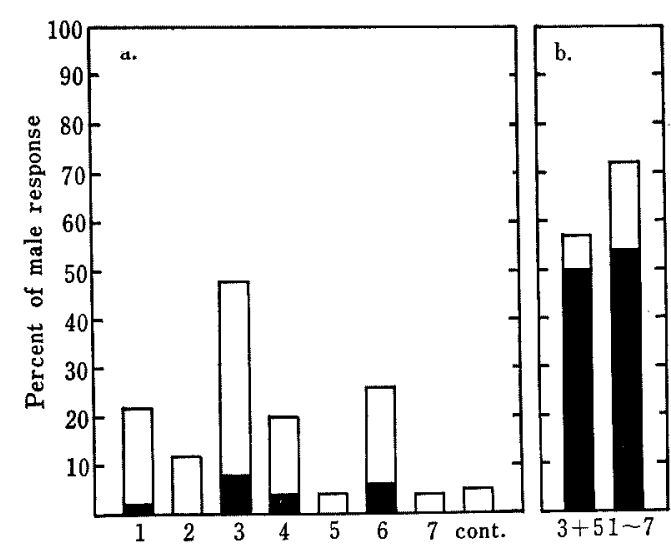

FIG. 1. Activity of Fractions Separated by GLC.

$\boldsymbol{Q}$, response of + : typical mating dance; $\square$, response of \pm : continuous walking or/and brief wing fluttering.

(a) individual fractions assayed with $5 \times 10^{-1}$ female equivalent.

(b) combined fractions assayed with $5 \times 10^{-2}$ female equivalent.

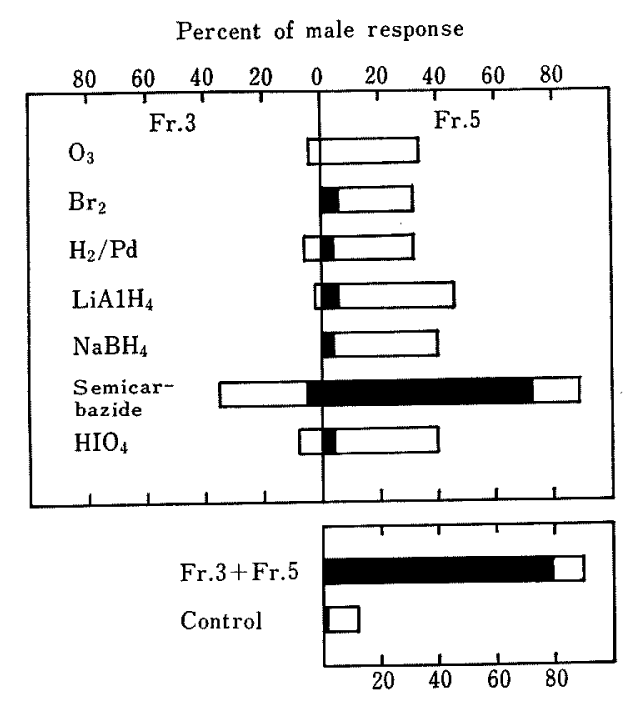

FIG. 2. Functional Group Tests for Fr. 3 and Fr. 5. Application of $5 \times 10^{-1}$ female equivalent.

was saponified with methanolic $2 \mathrm{~N}$-potassium hydroxide under reflux for $20 \mathrm{~min}$. Active material extracted from the reaction mixture with hexane was subjected to Florisil column chromatography, and active fraction eluted with $5 \%$ ether in hexane was further purified by silica gel thin layer chromatography, developed with benzene-hexane $(1: 1)$. Active material appeared in a fraction ( $R f$ values be- 
tween 0.4 and 0.5 ), which showed to contain several principal peaks by gas liquid chromatography with a column of $2.5 \mathrm{~m}$ by $3 \mathrm{~mm}$ (i.d.) of $1.5 \% \mathrm{OV}-17$ on Shimalite $\mathrm{W}$ in a Shimadzu GC-5A apparatus, when temperature was programmed from 150 to $200^{\circ} \mathrm{C}$ at $2^{\circ} \mathrm{C} / \mathrm{min}$. The principal peaks in the active fraction were fractionated by GLC. When each fraction was bioassayed, little or no activity was observed (Fig. 1). However, combining two fractions with principal peaks at retention times $13.0 \mathrm{~min}$ (Fr. 3) and $20.6 \mathrm{~min}$ (Fr. 5), retained the activity. These indicated that the pheromone of this species was consisted of at least two components.

Since the activity was only restored by combining Fr. 3 and Fr. 5, functional group tests of each fraction (Fig. 2) were carried out in combination with bioassay by adding an untreated fraction to another treated fraction with a reagent. Catalytic hydrogenation over Pd-C, treatments with bromine in carbon tetrachloride and ozone in 1,2-dichloroethane destroyed the activity of both fractions, which suggested the presence of a double bond or bonds. Lithium aluminum hydride and sodium borohydride reductions also destroyed the activity, which suggested the presence of an aldehyde, a ketone, or an epoxide group or groups. Moreover acid treatment with periodic acid destroyed the activity. These results indicated that the presence of an epoxide group or groups was suggested rather than an aldehyde or a ketone group. However unexpected result was obtained for Fr. 3, of which the activity was greatly retarded by treatment with semicarbazide in methanol.

Better purification for the active Florisil fraction was achieved by high speed liquid chromatography with a silica gel column of $25 \mathrm{~cm}$ by $2.1 \mathrm{~mm}$ (i.d.), developed with $2 \%$ dichloromethane in hexane in a Shimadzu-Du Pont 830 apparatus. Fractions were collected with every one minute and each fraction was monitored by GLC and bioassay. The retention indices of principal peaks contained in Fr. 3 (peak A) and Fr. 5 (peak B) were 1919 and 2117 on $\mathrm{OV}-17$ respectively, and these are very close to those of 1,2-epoxy-hexadecane (1917) and -octadecane (2123), which inclined to suggest that the pheromone components were the same class of compounds only differed by two methylene groups. In a gas liquid chromatography and mass spectroscopy on a $5 \%$ OV-17 column in a ShimadzuLKB 9000 apparatus, the mass spectrum of peak A exhibited a molecular ion peak at $m / e 238$, which was consistent with a molecular formula of $\mathrm{C}_{16} \mathrm{H}_{30} \mathrm{O}$ with double unsaturation. A principal fragment ion peak was at $m / e 220$ $\left(\mathrm{M}^{+}-\mathrm{H}_{2} \mathrm{O}\right)$, and no other appearent fragment ion peaks were observed except for groups of peaks spaced fourteen mass units apart and increased in abundance with decreasing mass. These suggested that peak A was a straight chain hydrocarbon with an epoxide and a double bond. This is consistent with the results of the functional group tests and gas chromatographic behavior. It was shown by GC-MS spectrum and mass fragmentography that peak $B$ was consisted of several components. Further analysis for Fr. 3 and purification of Fr. 5 are in due course.

Acknowledgement. The authors are grateful to Mr. T. Kitsuwa of Shimadzu Seisakusho Ltd. for measuring GC-MS spectra. Sincere thanks are also due to Professor N. Takahashi of Tokyo University and Dr. T. Tatsuno of this institute for much useful advice. This work was partly supported by grant of Science and Technology Agency.

\section{REFERENCES}

1) S. Tatsuki and J. Fukami, Kontyū, 40, 203 (1972).

2) S. Tatsuki, M. Kurihara, S. Atsusawa, K. Uchiumi, J. Fukami, and K. Kishino, Botyu-Kagaku, 40, 150 (1975).

3) K. K. Carrol, L. Lipid Res., 2, 135 (1961).

4) M. Jacobson, "Insect Sex Pheromone," Academic Press, Inc., New York, N.Y., 1972, p. 170. 